\title{
On-body Wearable Repeater as a Data Link Relay for In-body Wireless Implants
}

\author{
Sam Agneessens, Patrick Van Torre, Emmeric Tanghe, Günter Vermeeren, \\ Wout Joseph, Member IEEE and Hendrik Rogier, Senior Member, IEEE
}

\begin{abstract}
Wireless medical devices implanted at different locations in the human body have a wide application range. Yet, high data rate communication in the $2.4 \mathrm{GHz}$ Industrial, Scientific and Medical band suffers from high in-body attenuation loss. Link improvement cannot be obtained by simply increasing transmit power, as battery life is limited and in-body absorption has to remain low. To overcome these problems, a flexible on-body textile patch antenna, robustly matched directly to the human body, is designed and developed as part of a wearable repeater, enhancing communication with implanted wireless devices. This receive antenna, which can cope with different morphologies and patient movements, enables reliable high data rate and low power communication links with an implant. A data link measurement is performed for the on-body repeater system placed on the human torso, relaying the signals to nearby medical equipment, without wired connection to the patient. The performance of the data link is experimentally assessed in different measurement scenarios. For a repeater system relying on simple analog amplification, which is low cost, energy efficient and can be fully integrated into clothing, excellent results are obtained, with an average measured signal to noise ratio of $33 \mathrm{~dB}$ for tissue depths up to $85 \mathrm{~mm}$.
\end{abstract}

Index Terms - Textile antenna, on-body antenna, in-body radio channel, link budget, medical implants

\section{INTRODUCTION}

$\mathrm{W}$ ITH each passing day, the era of the internet of things draws closer. Wireless communication between virtually each conceivable device requires creative integration of antennas at unconventional locations. In the search of solutions, close-proximity, on-body and in-body antennas have received their fair share of attention.

On-body textile antennas boast the advantage of being flexible, light-weight and can easily be integrated unobtrusively into clothing. The range of applications for

$\begin{array}{lllll}\text { Manuscript } & \text { received } & 14 & \text { October }\end{array}$ S. Agneessens, P. Van Torre and H. Rogier are with the Department of Information Technology (INTEC), Ghent University, St. Pietersnieuwstraat 41, 9000 Ghent, Belgium. (E-mails: Sam.Agneessens@UGent.be, Patrick.VanTorre@UGent.be, Hendrik.Rogier@UGent.be)

E. Tanghe, G. Vermeeren and W. Joseph are with the Department of Information Technology, Interdisciplinary Institute for BroadBand Technology, Ghent University, Ghent B-9050, Belgium (e-mail: emmeric.tanghe@intec.ugent.be; $\quad$ gunter.vermeeren@intec.ugent.be; wout.joseph@intec.ugent.be).

P. Van Torre is also with Hogeschool Gent, INWE department, Valentin Vaerwyckweg 1, 9000 Ghent, Belgium which on-body textile antenna designs are proposed, is broad: WBAN communication [1], positioning [2], etc.

Research into implantable antennas is another hot topic. Wireless medical devices can be implanted almost everywhere in the human body, providing remote monitoring of patients by transmitting the collected biometric data to a receive station, which can help to temper the rising medical cost brought on by aging of the population. Such implantable antennas operate in the $402-405 \mathrm{MHz}$ Medical Implant Communication Services (MICS) band [3], the $2.45 \mathrm{GHz}$ Industrial, Scientific and Medical (ISM) band [4] or in multiple bands [5].

The antennas designed for the MICS band have limited bandwidth, making high data rates, required for high resolution applications, impossible. Another drawback of these low frequency antennas is that designs for the MICS band either may exhibit poor performance or too large dimensions to be implantable in a real life situation.

In many applications, $2.45 \mathrm{GHz}$ ISM band antennas are preferred: their size makes them suited for implantation, the good radiation performance allows for low power consumption, which is vital to achieve acceptable battery lifetime of the implanted devices. In addition, the larger bandwidth opens possibilities for high data rate applications, such as video transmission. The higher in-body attenuation, however, reduces the signal-to-noise (SNR) ratio more distinctly at $2.45 \mathrm{GHz}$ compared to the $402-405 \mathrm{MHz}$ MICS band, making it more difficult to set up reliable links at high data rates. Since in-body absorption needs to remain as low as possible, one cannot simply increase the transmitted power to maintain an acceptable SNR at the receiver (which cannot always be placed in the direct vicinity of the human body).

To overcome these conflicting demands, this letter presents an easy integrable, low-intrusion, flexible on-body repeater, leveraging textile materials to construct the radiating elements and safeguarding the patient's comfort, which is important in medical applications. The repeater topology consists of a transmit and receive antenna, as well as active circuitry.

The receiver antenna, which also operates in the $2.45 \mathrm{GHz}$ ISM band, differs from other textile antennas presented so far, as it is designed to be matched to and radiate into the human body, instead of radiating in free space away from the human body. Careful design guarantees stable antenna performance in changing conditions related to on-body use, such as people 
with different morphologies, a moving patient, variations in electrical parameters of different body parts, etc.

The active part of the repeater consists of an amplifier or transceiver, which transfers the received in-body signal to the transmitter, relaying it to a remote medical end unit for further processing. Parasitic oscillations in the repeater are avoided by using a textile ground plane to isolate the receiver from the transmitter. The goal of this letter is to present a wearable on-body repeater with experimentally validated performance for an in-to-out body link.

In Section II the antenna design related to the repeater is described. Section III presents data link measurements that include the wearable relay, leading to the conclusions presented in section IV.

\section{REPEATER'S RECEIVE ANTENNA DESIGN}

\section{A. Design}

As mentioned in Section I, the repeater needs to function in close vicinity of the human body under varying operating conditions, while providing maximum user comfort. On-body robustness can easily be achieved for the wearable transmit antenna by using a ground plane, extending at least $\lambda / 10$ along all sides of the antenna patch, to shield it from the patient. The repeater's receive antenna is more susceptible to the close proximity of the wearer as it is placed directly on the body, with its main beam pointing into the body, resulting in a more challenging design.

To fulfill these design goals, a rectangular ring topology with probe feed is adopted, which leverages textile materials to create a conformal, lightweight, and comfortable antenna, suited for on-body use. By exciting two orthogonal modes on the antenna patch, achieving wide bandwidth and allowing for circular polarization, which makes the orientation of the implanted in-body antenna much less critical.

The antenna substrate is a very flexible closed cell expanded rubber protective foam with permittivity $\varepsilon_{\mathrm{r}}=1.485$, a loss $\operatorname{tangent} \tan \delta=0.0243$ and a height $\mathrm{h}_{1}=3.94 \mathrm{~mm}$. A spacer, made from a foam layer of thickness $\mathrm{h} 2=7.92 \mathrm{~mm}$, is used to physically separate the conductive parts of the antenna from the body, providing steady performance by ensuring matching to $50 \Omega$ under changing conditions. Ground plane and patch are fabricated using the $80 \mu \mathrm{m}$ thick e-textile Flectron ${ }^{\circledR}$, which has a sheet resistivity of $0.18 \Omega / \mathrm{sq}$ at $2.45 \mathrm{GHz}$.

Using CST microwave studio, the antenna is optimized to cover the $2.4 \mathrm{GHz}$ ISM band (reflection coefficient, $\Gamma<-10 \mathrm{~dB}$ ) in distinctly different operating conditions: direct contact between antenna and body (on-body situation) and far away from the human body (free space situation), to ensure that the antenna remains reliable under varying operating conditions.

Parameters for the human tissue are chosen to be $\varepsilon_{\mathrm{r}}=50.8$ and $\sigma=2.01 \mathrm{~S} / \mathrm{m}$ at $2.45 \mathrm{GHz}$, corresponding to the tissue mimicking fluid MSL2450 recommended by CENELEC standard EN50383 [6] and used in Section III to fill the human phantom. Since size and shape of the human tissue were found to have small influence on the antenna resonance [3], instead of relying on a detailed body model, simulation time is decreased by simply positioning the antenna on a small homogeneous block (length and width $80 \mathrm{~mm}$, height $20 \mathrm{~mm}$ ) with dielectric properties corresponding to the MSL2450 fluid. This approach is validated in the next subsection by means of measurements.

Antenna topology and final dimensions are shown in Fig. 1.

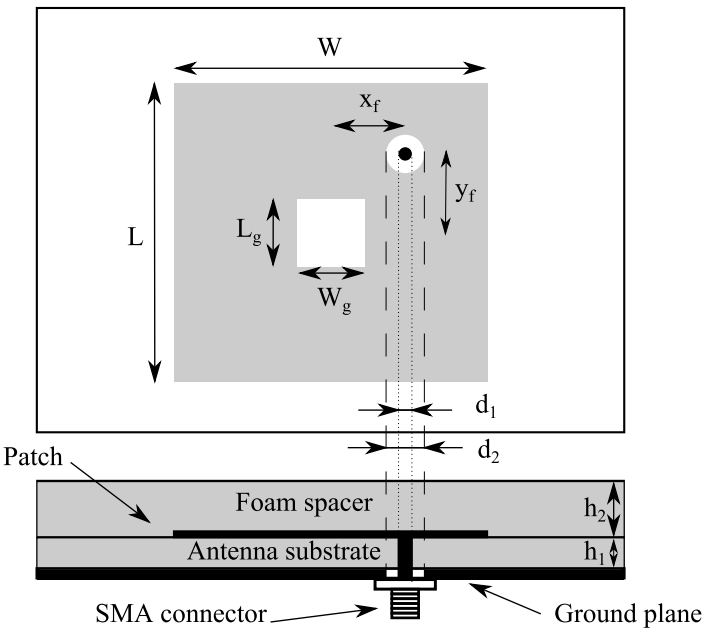

Fig. 1. Repeater-receive antenna topology. $(\mathrm{W}=40.9 \mathrm{~mm}, \mathrm{~L}=48.7 \mathrm{~mm}$, $\mathrm{W}_{\mathrm{g}}=8.8 \mathrm{~mm}, \mathrm{~L}_{\mathrm{g}}=13.2 \mathrm{~mm}, \mathrm{X}_{\mathrm{f}}=7.8 \mathrm{~mm}, \mathrm{Y}_{\mathrm{f}}=18.5 \mathrm{~mm}, \mathrm{~h}_{1}=3.94 \mathrm{~mm}$, $\mathrm{h}_{2}=7.92 \mathrm{~mm}, \mathrm{~d}_{1}=1.3 \mathrm{~mm}, \mathrm{~d}_{2}=5.5 \mathrm{~mm}$ )

\section{B. Simulation and measurement results}

Fig. 2. depicts the simulated and measured reflection coefficient when the antenna is placed on the oval ELI flat phantom (Speag, Zürich, Switzerland) filled with the MSL2450 liquid. The used flat phantom is compatible with the IEC 62209-2 standard and is used for compliance testing of handheld and body-mounted wireless devices in the frequency range of $30 \mathrm{MHz}$ to $6 \mathrm{GHz}$ at positions other than the ear. The antenna is well matched around the central frequency of $2.45 \mathrm{GHz}$ in both cases, and has a measured onbody bandwidth of $355 \mathrm{MHz}$, which is sufficient to cope with changes in the body parameters.

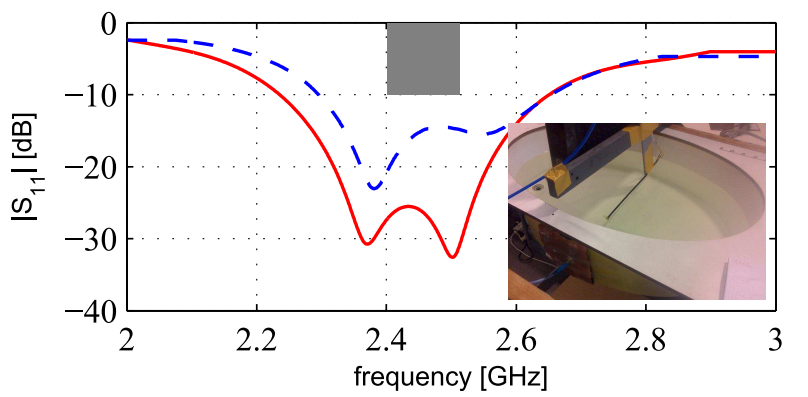

Fig. 2. Simulated (full line) and measured (dashed line) reflection coefficient for antenna placed on phantom filled with MSL2450 tissue mimicking liquid. Inset: on-body antenna placed on phantom.

As mentioned, no two subjects have the same morphology and dielectric properties are subject to large variations when considering different parts of the human body. Therefore, the 
antenna is tested on several distinctly different positions (stomach, back and head) on the body of a male subject with height $1.83 \mathrm{~m}$ and body mass index of 21.8. Fig. 3 demonstrates that the antenna remains well matched under these varying operating conditions, validating the antenna as a functional on-body antenna. We also tested the antenna on some different human subjects and the reflection curves are subject to some changes, but these are small and do not compromise the antennas good functionality

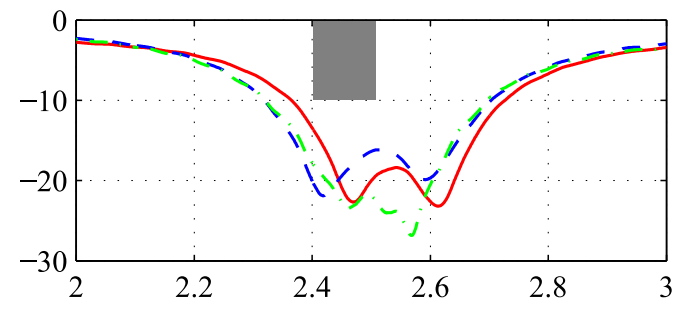

Fig. 3. Measured reflection coefficient for antenna placed at different positions on the human body: stomach (full line), back (dashed) and head (dot-dash).

\section{DATA LINK MEASUREMENT}

\section{A. Configuration}

To validate the improvement achieved by the use of an onbody repeater, we assess the data link quality between an implanted transmit antenna, placed at various depths in the human body, and a receiver. By means of a flat phantom, filled with tissue mimicking fluid MSL2450, the torso of a patient is emulated.

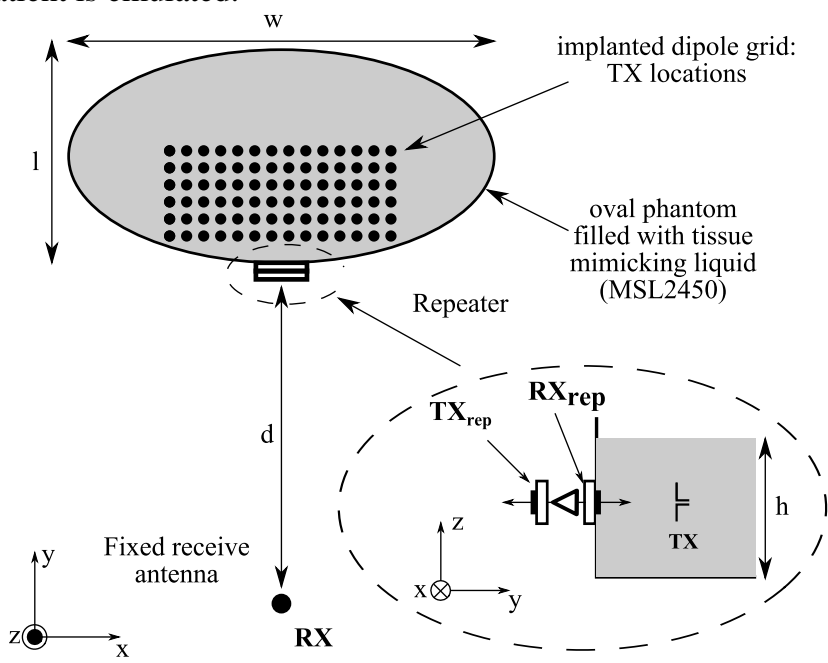

Fig. 4. Configuration: phantom filled with MSL2450 tissue mimicking liquid $\left(\varepsilon_{\mathrm{r}}=50.8, \sigma=2.01 \mathrm{~S} / \mathrm{m}\right.$ at $\left.2.45 \mathrm{GHz}\right)$, immersed dipole, repeater and fixed remote monitoring station. ( $\mathrm{w}=0.8 \mathrm{~m}, \mathrm{l}=0.5 \mathrm{~m}, \mathrm{~h}=0.12 \mathrm{~m}, \mathrm{~d}=2 \mathrm{~m}$ )

The general setup is shown in Fig. 4. Different implant positions, varying from $-10 \mathrm{~cm}$ to $10 \mathrm{~cm}$ along the $\mathrm{x}$-axis with respect to the repeater's receiver placed at the origin, and depths, ranging from $1 \mathrm{~cm}$ to $10 \mathrm{~cm}$ (y-axis), are realized by a vertically placed in-body isolated dipole antenna [7], which is moved by a scanning system and has a measured gain of 8.7 $\mathrm{dBi}$ in the MSL2450 liquid. The repeater is placed on the side of the phantom, comparable to placement on the human torso, with the receive $\mathrm{RX}_{\text {rep }}$ antenna's boresight along the y-axis and the direction of main gain of the transmit $\mathrm{TX}_{\text {rep }}$ antenna, described in [8], along the negative y-axis. The latter antenna is linearly polarized along the $\mathrm{z}$-axis, has $8.2 \mathrm{dBi}$ gainand is used to relay the data to a third remote antenna, being an external monitoring device placed at a fixed location $\mathrm{d}=2 \mathrm{~m}$ away in line-of-sight of the phantom body.

Data modulation, transmission and remodulation is handled by the Signalion Hardware in the Loop (HaLo) equipment, a Multiple Input Multiple Output (MIMO) test system described in [8]. The transmit power is $10 \mathrm{~mW}$, which corresponds to about half the allowed SAR limit of $2 \mathrm{~W} / \mathrm{kg}$ averaged over 10 $\mathrm{g}$ of tissue [IEC 62209-1]. The HaLo hardware measures the SNR by using a transmit gap after each sent frame. During this transmit gap, the noise, measured by the receiver, is sampled in order to calculate the noise power.

\section{B. Measurement scenario's and results}

Scenario 1: Direct link from implanted antenna (TX) to medical end terminal $(R X)$

The first scenario represents a patient in the same room as a wireless monitoring station. An implanted device gathers data, which is transmitted wirelessly to a remote terminal, placed at a distance $\mathrm{d}=2 \mathrm{~m}$. As the patient's movements are not constrained by wires maximal comfort is achieved. The received SNR in this first test has an average of $8 \mathrm{~dB}$ over the scanning grid, which only allows for low data rate communications. Note that signal paths leaving the phantom along top and bottom (where liquid depth is lower) significantly contribute to propagation, which may result in a constant, and higher than expected, SNR for larger depths.

Scenario 2: Direct on-body reception

Fig. 5 displays the measurement results for the reception by the on-body antenna $\left(\mathrm{RX}_{\mathrm{rep}}\right)$, indicating an average SNR of $30.1 \mathrm{~dB}$. The SNR now remains larger than $15 \mathrm{~dB}$ for depths up to $85 \mathrm{~mm}$ in the body, which is a considerable improvement over the direct link scenario, allowing for highrate data communications, as required for neuro-electronic interfaces or video links for capsule endoscopy applications, which are not achievable by means of the direct link scenario.

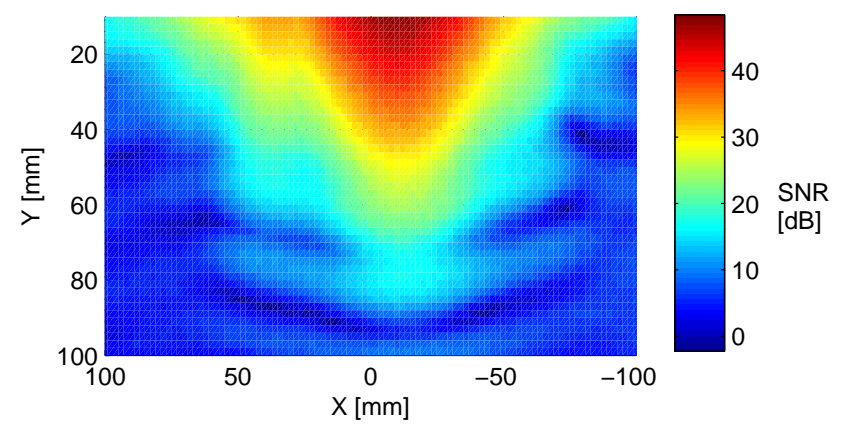

Fig. 5. Scenario 2: Received SNR on on-body antenna $\left(\mathrm{RX}_{\mathrm{rep}}\right)(\mathrm{Y}$-axis $=$ antenna depth in body; $\mathrm{X}$-axis $=$ position relative to the on-body antenna). 
Scenario 3: On-body transceiver

Attachment of the receiving device directly on-body reduces the wearability, patient's movements and hence comfort. To overcome this, the third scenario uses a wireless transceiver, which demodulates the signal, received by $\mathrm{RX}_{\text {rep}}$, and remodulates it for transmission via antenna $\mathrm{TX}_{\text {rep }}$, resulting in a compact wearable module suitable for unobtrusive integration [8]. Measurement results demonstrate an average SNR of $40 \mathrm{~dB}$, which does not introduce bit errors in a 2 Mbit/s QPSK test transmission. However, full transceiver hardware and signal processing are required, which is expensive, large and power consuming.

Scenario 4: Retransmission with amplification

A simpler scenario consists of retransmitting the received signal, without data processing. An amplifier, with $43.8 \mathrm{~dB}$ gain at $2.45 \mathrm{GHz}$, is used to amplify the signal received by $\mathrm{RX}_{\text {rep}}$, which is then retransmitted by $\mathrm{TX}_{\text {end }}$. Oscillations are avoided by the shielding effect of the textile ground planes. Measured isolation between $\mathrm{RX}_{\text {rep }}$ and $\mathrm{TX}_{\text {rep }}$ is $55 \mathrm{~dB}$. Received SNR levels are displayed in Fig. 6. The SNR values achieved at remote terminal are very comparable to the results achieved in the second scenario (direct on-body reception), with an average SNR of $33.0 \mathrm{~dB}$. Due to interference between the direct and retransmitted signal, some side lobes are visible for small depths of the implanted antenna. This effect is not expected to cause significant problems.

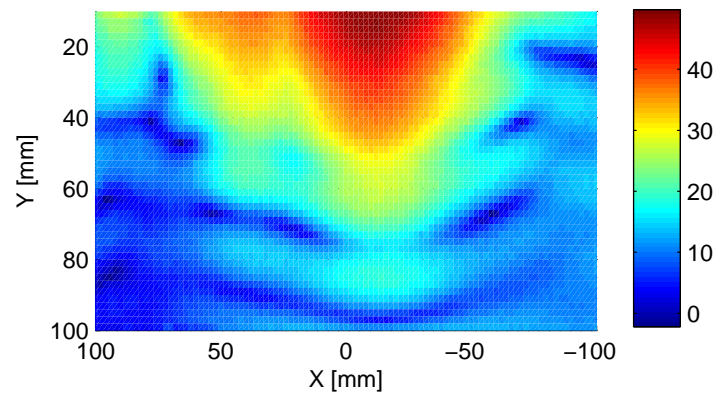

Fig. 6. Scenario 4: Received SNR on RX, retransmitted by amplification (Yaxis $=\mathrm{TX}$ depth in body; $\mathrm{X}$-axis $=$ position relative to $\mathrm{RX}_{\text {rep }}$ antenna's center).

\section{CONCLUSION}

An on-body receive antenna for communications with wireless implants was designed, fabricated and tested. The design is robust (i.e. it can cope with different morphologies, patient movements, etc.) and is well suited for efficient wireless communication with implanted devices, allowing lower transmit power and energy conservation for an implanted battery-powered device.

Measurements using a tissue mimicking liquid, show a considerable improvement with respect to the case without an on-body receiver with received signal levels of $15 \mathrm{~dB}$ SNR for tissue depths up to $85 \mathrm{~mm}$, enabling high data-rate links.

A transceiver system with full re-modulation of data is tested and validated as a liable substitute for the often cumbersome wired connection between patient and medical end device, improving patient comfort. A repeater system making use of simple analog amplification works very reliably and is, in comparison to the transceiver set up, very low cost, energy efficient, and can be fully integrated into clothing. Measurements confirm the high signal-to-noise ratios for the relayed signal, received at $2 \mathrm{~m}$ from the body.

Future research will focus on further link improvement by exploiting multiple antennas, diversity and MIMO techniques.

\section{ACKNOWLEDGMENT}

The authors would like to thank Sioen Industries for their support and the Belgian Science Policy Office (IUAP Program) and the FWO-V (Research Foundation - Flanders) for their financial support.

\section{REFERENCES}

[1] A. Tronquo, H. Rogier, C. Hertleer and L. V. Langenhove, "Robust planar textile antenna for wireless body LANs operating in $2.45 \mathrm{GHz}$ ISM band," Electronics Letters, vol. 42, no. 3, pp. 142-143, 2006.

[2] L. Vallozzi, W. Vandendriessche, H. Rogier, C. Hertleer and M. L. Scarpello, "Wearable textile GPS antenna for integration in protective garments," in 2010 Proceedings of the Fourth European Conference on Antennas and Propagation (EuCAP), Barcelona, Spain, 2010.

[3] A. Kiourti and K. Nikita, "Miniature Scalp-Implantable Antennas for Telemetry in the MICS and ISM Bands: Design, Safety Considerations and Link Budget Analysis," IEEE Transactions on Antennas and Propagation, vol. 60, no. 8, pp. 3568-3575, 2012.

[4] M. Scarpello, D. Kurup, H. Rogier, D. V. Ginste, F. Axisa, J. Vanfleteren, W. Joseph, L. Martens and G. Vermeeren, "Design of an Implantable Slot Dipole Conformal Flexible Antenna for Biomedical Applications," IEEE Transactions on Antennas and Propagation, vol. 59, no. 10, pp. 35563564, 2011.

[5] F.-J. Huang, C.-M. Lee, C.-L. Chang, L.-K. Chen, T.-C. Yo and C.-H. Luo, "Rectenna Application of Miniaturized Implantable Antenna Design for Triple-Band Biotelemetry Communication," IEEE Transactions on Antennas and Propagation, vol. 59, no. 7, pp. 2646-2653, 2011.

[6] CENELEC, "EN50383: basic standard for the calculation and measurement of electromagnetic field strength and SAR related to human exposure from radio base stations and fixed terminal stations for wireless telecommunication systems (110 MHz - $40 \mathrm{GHz})$," September 2012.

[7] D. Kurup, W. Joseph, G. Vermeeren and L. Martens, "Inbody Path Loss Model for Homogeneous Human Tissues," IEEE Transactions On Electromagnetic Compatibility, vol. 54, no. 3, pp. 556-564, 2012.

[8] L. Vallozzi, P. V. Torre, C. Hertleer, H. Rogier, M. Moeneclaey and J. Verhaevert, "Wireless Communication for Firefighters Using Dual-Polarized Textile Antennas Integrated in Their Garment," IEEE Transactions on Antennas and Propagation, vol. 58, no. 4, pp. 1357-1368, 2010 . 